\title{
SPACES OF COUNTABLE AND POINT-COUNTABLE TYPE
}

\author{
BY \\ J. E. VAUGHAN
}

\begin{abstract}
These spaces were introduced by M. Henriksen and J. R. Isbell, and A. V. Arhangel'skiir, who proved results about the placement of such spaces in their compactifications. In the present paper, these results are consolidated using new terminology. In addition, further results concerning the heredity of these spaces are obtained. Generalizations of these spaces are introduced, and an analogous treatment is given for them. Finally, some examples are given of which one gives a solution to a problem raised by Arhangel'skin by showing that the perfect image of a first countable space need not be of point-countable type.
\end{abstract}

1. Introduction. This paper is concerned with the spaces mentioned in the title and generalizations of them. The class of spaces of countable type was introduced by Henriksen and Isbell [5] under the name Lindelöf at infinity. The class of spaces of point-countable type was introduced by Arhangel'skii in [1], and he made a long study of both classes of spaces in [2].

These spaces can be easily defined. A topological space is of countable (resp. point-countable) type provided every compact set (resp. point) in the space is contained in a compact set of countable character. Recall that the character of a subset of a space (=topological space) is the smallest cardinal for which there exists a fundamental system of neighborhoods at the subset. Most of the results in this paper are related to the placement of a Tychonoff space (=completely regular and $T_{1}$ ) in its compactifications as given in the following theorem (the proof of which is known in the literature). Let $\beta X$ be the Stone-Čech compactification of $X$.

THEOREM 1. Let $X$ be a Tychonoff space.

(a) $X$ is locally compact $\Leftrightarrow X$ is open in $\beta X \Leftrightarrow \beta X-X$ is compact.

(b) $X$ is complete $\Leftrightarrow X$ is $a G_{\delta}$ in $\beta X \Leftrightarrow \beta X-X$ is $\sigma$-compact.

(c) $X$ is of countable type $\Leftrightarrow X$ is a generalized $G_{\delta}$ in $\beta X \Leftrightarrow \beta X-X$ is Lindelöf.

(d) $X$ is of point-countable type $\Leftrightarrow X$ is a point-generalized $G_{\delta}$ in $\beta X \Rightarrow \beta X-X$ is real-compact.

Presented to the Society, August 27, 1969 under the title On spaces of countable and pointwisecountable type; received by the editors August 26, 1969.

AMS Subject Classifications. Primary 5452, 5453, 5460.

Key Words and Phrases. Spaces of countable type, spaces of point-countable type, Tychonoff spaces, character of a set, pseudocharacter of a set, compactifications, Stone-Čech compactifications, generalized $F_{\sigma}, Q$-closed, real-compact.

Copyright (C) 1970, American Mathematical Society 
In $\S 2$ we will define and elaborate the notions of generalized $G_{\delta}$ and pointgeneralized $G_{\delta}$ and give an application (2.9) of Theorem 1. In $\S 3$ we shall give the references which are needed to prove Theorem 1 , and give a new proof to a theorem of Mrówka. In $\$ 4$ an example is given to show that " $\beta X-X$ is real-compact" is not equivalent to the other statements in Theorem 1 (d). Some results on the heredity of these spaces are given in $\$ 5$, and in $\$ 6$ we consider the consequence of replacing "character" by "pseudocharacter" in the definitions given above. Some examples are given in \$7. In particular, Example 7.5 solves the problem raised by Arhangel'skii in [2, Problem 3, p. 60] by showing that the perfect image of a first countable space need not be of point-countable type.

2. Generalized $G_{\delta}$, point-generalized $G_{\delta}$, and $Q$-closed sets. The origin of the first of these notions dates back at least to Smirnov [10] where he defined a set $A$ to be normally placed in a space $X$ provided for every open set $U$ containing $A$, there exists a set $F$ which is an $F_{\sigma}$ in $X$ and $A \subset F \subset U$. Later Michael [6, p. 836] used the terminology generalized $F_{\sigma}$ instead of normally placed. One of Smirnov's main results in [10] is

2.1 Theorem (Smirnov). Let $X$ be a Tychonoff space. If $X$ is a generalized $F_{\sigma}$ in a Lindelöf space, then $X$ is Lindelöf. If $X$ is a Lindelöf space, then $X$ is a generalized $F_{\sigma}$ in every space which contains it.

The concept of generalized $F_{\sigma}$ leads one to define the dual notion. We say that a set $B$ is a space $X$ is a generalized $G_{\delta}$ in $X$ provided $X-B$ is a generalized $F_{\sigma}$ in $X$.

2.2. Proposition. Let $X$ be a space and $B \subset X . B$ is a generalized $G_{\delta}$ in $X$ if and only if for every subset $H$ of $B$ which is closed in $X$, there exists a $G_{\delta}$-set $G$ in $X$ such that $H \subset G \subset B$.

Clearly, every $G_{\delta}$ is a generalized $G_{\delta}$, and a closed generalized $G_{\delta}$ is a $G_{\delta}$, but not every generalized $G_{\delta}$ is a $G_{\delta}$. See 2.8.

A natural way to weaken the concept of generalized $G_{\delta}$ is to work with points instead of closed sets in 2.2. Call a subset $A$ of a space $X$ a point-generalized $G_{\delta}$ in $X$ provided for every $x \in A$ there exists a $G_{\delta}$-set $G$ in $X$ such that $x \in G \subset A$. In a $T_{1}$ space, every generalized $G_{\delta}$ is a point-generalized $G_{\delta}$. In a space where every closed set (resp. point) is a $G_{\delta}$, every subset is a generalized (resp. point-generalized) $G_{\delta}$, but not every point-generalized $G_{\delta}$ is a generalized $G_{\delta}$. See 2.8 .

The dual notion to point-generalized $G_{\delta}$ was introduced by Mrówka [7]. He called the concept $Q$-closed (rather than point-generalized $F_{\sigma}$ ), and defined a set $A$ in a space $X$ to be $Q$-closed in $X$ provided for every $x \notin A$ there exists a $G_{\delta}$-set $G$ in $X$ such that $x \in G$ and $G \cap A=\varnothing$. An important result in [7] is

2.3. THEOREM (MRÓWKA). A Tychonoff space $X$ is real-compact if and only if $X$ is $Q$-closed in its Stone-Cech compactification.

The next two propositions can be easily proved. 
2.4. Proposition. Let $X$ be a space and $A \subset X . A$ is $Q$-closed in $X$ if and only if $X-A$ is a point-generalized $G_{\delta}$ in $X$.

2.5. Proposition. Let $X$ be a space and $A \subset X$. The following are equivalent.

(a) $A$ is a point-generalized $G_{\delta}$ in $X$.

(b) $A$ is a union of point-generalized $G_{\delta}$-sets in $X$.

(c) $A$ is a union of $G_{\delta}$-sets in $X$.

Further, if $X$ is a $T_{1}$-space, the following statement is equivalent to the above three:

(d) $A$ is a union of generalized $G_{\delta}$-sets in $X$.

2.6. Proposition (Transitive law). Let $Z$ be a space and $X \subset Y \subset Z$. If $X$ is a generalized (resp. point-generalized) $G_{\delta}$ in $Y$ and $Y$ is a generalized (resp. pointgeneralized) $G_{\delta}$ in $Z$, then $X$ is a generalized (resp. point-generalized) $G_{\delta}$ in $Z$.

Proof. We prove this for the case of generalized $G_{\delta}$. Let $H \subset X$ be such that $H$ is closed in $Z$. Then $H \subset Y$ so there exist open sets $G_{i}$ in $Z$ such that $H \subset \bigcap_{i=1}^{\infty} G_{i} \subset Y$. Further, $H$ is closed in $Y$, hence there exist sets $V_{i}$ open in $Y$ such that $H \subset$ $\bigcap_{i=1}^{\infty} V_{i} \subset X$. Let $V_{i}^{\prime}$ be open in $Z$ such that $V_{i}^{\prime} \cap Y=V_{i}$, then set

$$
G=\bigcap_{i=1}^{\infty}\left(V_{i}^{\prime} \cap G_{i}\right)
$$

and note $H \subset G \subset X$.

The terminology for compactification used in the next proposition can be found in $[3, \S 4$, p. 125$]$. In particular, $(\beta X, \beta)$ is the Stone-Čech compactification of $X$ where $\beta: X \rightarrow \beta X$ is a homeomorphic embedding of $X$ into the compact space $\beta X$.

2.7. Proposition. Let $X$ be a Tychonoff space. The following are equivalent.

(a) For every compactification $(Y, c)$ of $X, c(X)$ is a generalized (resp. pointgeneralized) $G_{\delta}$ in $Y$.

(b) $\beta(X)$ is a generalized (resp. point-generalized) $G_{\delta}$ in $\beta X$.

(c) There exists a compactification $(Y, c)$ of $X$ such that $c(X)$ is a generalized (resp: point-generalized) $G_{\delta}$ in $Y$.

Proof. The proof is similar to that of [3, Theorem 1, p. 142] and 6.1.

2.8. EXAMPLES. (a) Let $I$ be the closed unit interval and $Q$ the set of rational points in $I$. It is well known that $Q$ is not a $G_{\delta}$ in $I$, but since $I$ is perfectly normal, $Q$ is a generalized $G_{\delta}$ in $I$.

(b) Let $I_{0}$ be the closed unit interval with the half open (on the left) interval topology. Let $M$ be the union of the top and bottom lines of the lexicographically ordered square, then the bottom of the square is $I_{0}$ and as above $I_{0}$ is a generalized $G_{\delta}$ in $M$ which is not a $G_{\delta}$.

(c) Let $X$ be the lexicographically ordered square. It is a compact, first countable, Hausdorff space. Let $D$ be the set of all points in $X$ whose second coordinate is rational. Then $D$ is dense in $X$ and contains $M$ : the union of the top and bottom lines. Of course, $D$ is a point-generalized $G_{\delta}$ in $X$, but it is not a generalized $G_{\delta}$ 
in $X$. To see this consider $M \subset D$. Since $M$ is compact, it is closed in $X$. Suppose there exist open sets $G_{i}$ in $X$ such that $M \subset \bigcap_{i=1}^{\infty} G_{i} \subset D$. Then $X-D \subset \bigcup_{i=1}^{\infty}\left(X-G_{i}\right)$ $\subset X-M$. Each $X-G_{i}$ is a compact set which misses $M$. Hence, each $X-G_{i}$ is contained in a finite number of vertical lines. Thus $\bigcup_{i=1}^{\infty} X-G_{i}$ is contained in a countable number of vertical lines, and so this set cannot contain $X-D$.

The results in this paragraph can be combined with Theorem 1 and 2.7 to give some examples.

2.9. ExAmples. (a) Since $Q$ is dense in the compact set $I$, and is a generalized $G_{\delta}$ in $I$ but not a $G_{\delta}$ in $I$ we have that $Q$ is a space of countable type but not a complete space.

(b) In a similar manner, $I_{0}$ is a nonmetric space of countable type which is not a complete space.

(c) In a similar manner, $D$ is a first countable space (hence of point-countable type) which is not of countable type. Also see 7.4.

The first two examples are known, but the third appears to be new. In fact, no space of point-countable type-not of countable type-was given in [1] or [2].

3. Proof of Theorem 1. For the remainder of this paper, we shall usually assume that $X \subset \beta X$.

The first equivalence in part (a) is well known, and the second is trivial. The first equivalence in part (b) is the definition of complete space, and the second is trivial. The proof of part (c) follows from [5, Theorem 3.6, p. 98] which states that a space $X$ is of countable type if and only if $\beta X-X$ is Lindelöf, and from Theorem 2.1. The first equivalence in (d) follows at once from [2, Theorem 3.13, p. 43] which states that a space $X$ is of point-countable type if and only if $X$ is a union of $G_{\delta}$-sets in $\beta X$, and from 2.5. Finally, by $2.4, X$ is a point-generalized $G_{\delta}$ in $\beta X$ implies that $\beta X-X$ is $Q$-closed in $\beta X$. That $\beta X-X$ is real-compact follows at once from [7, Theorem 2, p. 950] which states that a $Q$-closed subset of a realcompact space is real-compact.

The preceding proof has two points which deserve further comments. First, it would be desirable to have a direct proof of the first equivalence of part (c) which would fit into the theory of spaces of countable type as developed by Arhangel'skir in [2]. A corollary to that result and 2.1 is [5, Theorem 3.6, p. 98] which was used above to prove part (c). Secondly, [7, Theorem 2, p. 950] which was used in the proof of part (d) was itself proved by measure-theoretic techniques. Below we give a shorter proof which uses only topological notions.

Alternate proof of the first equivalence in part (c).

3.1. LEMMA. Let $H$ be a compact subset of a space $X$ which is itself dense in $a$ space $Y$. Then $H$ is of countable character in $X$ if and only if $H$ is of countable character in $Y$.

Proof. This is a special case of [5, Lemma 3.5, p. 98]. 
If $X$ is a generalized $G_{\delta}$ in $\beta X$, and $H$ is a compact subset of $X$, then $H$ is compact and hence closed in $\beta X$. Thus, there exist open sets $G_{i}$ in $\beta X$ such that $H \subset \bigcap_{i=1}^{\infty} G_{i}$ $\subset X$. Construct open sets $V_{i}$ in $\beta X$ such that $H \subset V_{i} \subset \bar{V}_{i} \subset V_{i-1} \cap G_{i}$ where $V_{0}=\beta X$. Then we have $K=\bigcap_{i=1}^{\infty} V_{i}=\bigcap_{i=1}^{\infty} \bar{V}_{i} \subset \bigcap_{i=1}^{\infty} G_{i}$, so $K$ is a compact $G_{\delta}$ in $\beta X$ which is a subset of $X$. It is well known that a closed $G_{\delta}$ in a compact space is of countable character. Hence $K$ is of countable character in $\beta X$, and a fortiori in $X$.

If $X$ is a space of countable type, and $H$ is a subset of $X$ which is closed in $\beta X$, then $H$ is compact. Hence there exists a compact set $K \subset X$ of countable character in $X$ which contains $H$. By $3.1, K$ is of countable character in $\beta X$. In particular $K$ is a $G_{\delta}$ in $\beta X$ and $H \subset K \subset X$. This completes the proof.

A topological proof of [7, Theorem 2, p. 950]: A $Q$-closed subset of a realcompact space is real-compact. Let $A$ be a $Q$-closed subset of a real-compact space $X$. Since $A$ is $Q$-closed in $\bar{A}$ and $\bar{A}$ is real-compact, we may assume that $A$ is dense in $X$. If we let $j: A \rightarrow \beta X$ be the inclusion map, then we have that $(A, j)$ is a compactification of $A$. It is easy to verify the "transitive law" similar to 2.6 for $Q$-closed sets; so we have $j(A)$ is $Q$-closed in $\beta X$. Let $(\beta A, \beta)$ be the StoneČech compactification of $A=\beta(A)$. We now show that $A$ is $Q$-closed in $\beta A$. Let $f: \beta A \rightarrow \beta X$ be the continuous map such that $j=f \circ \beta$. Let $p \in \beta A-A$, then $f(p)$ $\in \beta X-j(A)$ [3, Theorem 7, p. 128]. Thus there exists a $G_{\delta}$-set $G$ in $\beta X$ such that $f(p) \in G$ and $G \cap j(A)=\varnothing$. It follows that $f^{-1}(G)$ is a $G_{\delta}$ in $\beta A$ which contains $p$ and misses $A$. The result follows from 2.3.

4. An example of a space $X$ which is not of point-countable type, and $\beta X-X$ is real-compact (under the assumption of the continuum hypothesis). This example is a combination of a construction by W. Rudin [9, p. 415] and a construction given in [3, Example 3, p. 133] which yields the following lemma. Let $N$ be the natural numbers, and recall that a point is called a $P$-point if every $G_{\delta}$ containing the point is a neighborhood of the point.

4.1. Lemma [CH]. There exists a discrete subset $R$ of $\beta N-N$ which has the cardinality of the continuum $\mathrm{c}$, and in the space $\beta N-N$ has a P-point as an accumulation point.

If we assume the lemma is true, then $X=\beta N-R$ is the desired example. To see this, first note $N \subset X$ hence $\beta X=\beta N$ and $\beta X-X=R$ which is real-compact [4, pp. $163,165]$. Let $p \in X$ be a $P$-point which is an accumulation point of $R$. It is easy to see that every $G_{\delta}$ in $X$ which contains $p$ must also hit $R$. Thus $\beta X-X$ is not $Q$-closed in $\beta X$, so $X$ is not of point-countable type.

Proof of 4.1. Let $\Omega$ be the first uncountable ordinal. Rudin [9, p. 414] proved that there exist exactly $c$ open-closed sets in $\beta N-N$ and that these sets form a base for the topology of $\beta \boldsymbol{N}-\boldsymbol{N}$. By the continuum hypothesis we may assume these sets are $\left\{W_{\alpha}: \alpha<\Omega\right\}$ and that $W_{0}=\beta N-N$. Let $A_{0}$ and $B_{0}$ be any two disjoint nonempty open-closed sets in $\beta N-N$. Assume we have constructed pairs of sets $\left\{\left(A_{\beta}, B_{\beta}\right): \beta<\alpha\right\}$ such that for every $\gamma<\alpha$ the following hold: 
(1) $E_{\gamma}=\bigcap_{\beta \leqq \gamma} A_{\beta} \neq \varnothing$.

(2) $A_{\beta} \cap B_{\beta}=\varnothing$ if $\beta \leqq \gamma$.

(3) $B_{\beta} \subset A_{\delta}$ if $\delta<\beta \leqq \gamma$.

We now construct a new pair $\left(A_{\alpha}, B_{\alpha}\right)$. First note $\bigcap\left\{E_{\gamma}: \gamma<\alpha\right\}=\bigcap\left\{A_{\gamma}: \gamma<\alpha\right\}$ $\neq \varnothing$ and hence by $[9$, Theorem 3.3, p. 414] contains a nonempty open-closed set $C_{\alpha}$. Let $D_{\alpha}=C_{\alpha}$ if $C_{\alpha} \cap W_{\alpha}=\varnothing$ and $D_{\alpha}=C_{\alpha} \cap W_{\alpha}$ if $C_{\alpha} \cap W_{\alpha} \neq \varnothing$. Let $A_{\alpha}$ and $B_{\alpha}$ be any nonempty disjoint open-closed subsets of $D_{\alpha}$. The collection

$$
\left\{\left(A_{\beta}, B_{\beta}\right): \beta \leqq \alpha\right\}
$$

satisfies (1), (2) and (3) above. By transfinite induction we have a collection $\left\{A_{\alpha}: \alpha<\Omega\right\}$ which is a fundamental system of neighborhoods for a $P$-point $p$ in $\beta N-N$. The proof of this is the same as the proof of [9, Theorem 4.2, p. 415]. Further $\left\{B_{\alpha}: \alpha<\Omega\right\}$ is a collection of nonempty mutually disjoint open-closed subsets of $\beta N-N$. Let $x_{\alpha} \in B_{\alpha}$, all $\alpha<\Omega$, and set $R=\left\{x_{\alpha}: \alpha<\Omega\right\}$. Then $R$ is a discrete subset of $\beta N-N$ and has the $P$-point $p$ as accumulation point.

Added in proof. After this paper was submitted for publication, a note by $S$. Negrepontis [11] appeared in which he constructed for another purpose the set $R$ of 4.1.

5. Hereditary properties. In this paragraph we make use of the placement of a space $X$ in its Stone-Čech compactification as given in Theorem 1 .

5.1. Lemma. Let $Z$ be a space, and $X \subset Y \subset Z$, where $Y$ is a generalized (resp. point-generalized) $G_{\delta}$ in $Z$. (a) If $X$ is closed in $Y$, then $X$ is a generalized (resp. point-generalized) $G_{\delta}$ in $\mathrm{Cl}_{z} X$. (b) If $X$ is a generalized (resp. point-generalized) $G_{\delta}$ in $Y$, then $X$ is a generalized (resp. point-generalized) $G_{\delta}$ in $\mathrm{Cl}_{z} X$.

Proof. We prove this for the case of generalized $G_{\delta}$. (a) Since $X$ is closed in $Y$, we know $Y \cap \mathrm{Cl}_{z} X=X$. If $H \subset X \subset Y$ and $H$ is closed in $\mathrm{Cl}_{Z} X$, then $H$ is closed in $Z$. By the hypothesis on $Y$, there exists a $G_{\delta}$-set $G$ in $Z$ such that $H \subset G \subset Y$. Hence, $H \subset G \cap \mathrm{Cl}_{z} X \subset Y \cap \mathrm{Cl}_{z} X=X$. (b) By 2.6,X is a generalized $G_{\delta}$ in $Z$, and clearly in any intermediate closed set.

Arhangel'skir proved $\left[2\right.$, p. 44] that closed and $G_{\delta}$ subsets of spaces of pointcountable type are of the same type. Similarly we have

5.2. Proposition. Let $Y$ be a Tychonoff space of countable type. Every closed subset and every generalized $G_{\delta}$-subset of $Y$ are also spaces of countable type.

Proof. By Theorem 1, $Y$ is a generalized $G_{\delta}$ in $\beta Y$. If $X$ is closed or a generalized $G_{\delta}$ in $Y$, then by $5.1, X$ is a generalized $G_{\delta}$ in $\mathrm{Cl}_{\beta Y} X$. By 2.7 , we have $X$ is a generalized $G_{\delta}$ in $\beta X$, so $X$ is of countable type.

A similar proof can be given for the following generalization of [2, Corollary, p. 44] mentioned above. 
5.3. Proposition. Let $Y$ be a Tychonoff space of point-countable type. If $X$ is a point-generalized $G_{\delta}$ in $Y$, then $X$ is of point-countable type.

5.4. Proposition. Let $Y$ be a Tychonoff space in which every closed set (resp. point) is a $G_{\delta}$. If $Y$ is of countable (resp. point-countable) type, then every subspace of $Y$ is of the same type.

Proof. If $X \subset Y$, then $X$ is a generalized (resp. point-generalized) $G_{\delta}$ in $Y$. The result follows from 5.2 and 5.3.

As a corollary to the preceding result, we get [5, Theorem 3.9, p. 99] and a similar result below for the point-countable case.

5.5. Corollary. Let $Y$ be a Tychonoff space which is hereditarily Lindelöf. If $Y$ is of countable (resp. point-countable) type, then every subspace is of the same type.

5.6. Corollary. Let $Y$ be a Tychonoff space in which every point is a $G_{\delta}$. If $Y$ is of point-countable type, then every subspace of $Y$ is of the same type.

In conclusion of this paragraph, it should be noted that these properties are not hereditary to arbitrary subspaces.

6. Spaces of pseudocountable and pseudopoint-countable type. Recall that the pseudocharacter of a set in a topological space is the smallest cardinal $\mathfrak{m}$ for which the set is an intersection of $\mathfrak{m}$ open sets. A natural way to generalize the notion of spaces of countable and point-countable type is to replace "character" by "pseudocharacter" in their definitions: A space is said to be of pseudocountable (resp. pseudopoint-countable) type provided every compact set (resp. point) is contained in a compact set of countable pseudocharacter, i.e., a compact $G_{\delta}$.

Arhangel'skir [2, Theorem $3.7^{\prime}$, p. 37] proved that the spaces of countable and point-countable type are $k$-spaces. The same does not hold for their "pseudo" generalization (7.1). The methods of the preceding sections, however, can be applied to characterize these spaces by their placement in their compactifications, and to obtain some results concerning their hereditary properties. Also we shall consider the behavior of these spaces under products and perfect mappings.

Fo see how these spaces are placed in their compactifications, it is necessary to consider the following definitions. A set $A$ in a topological space $X$ is called a pseudogeneralized $G_{\delta}$ (resp. pseudopoint-generalized $G_{\delta}$ ) in $X$ provided for every $H \subset A$ such that $H$ is closed in $X$ (resp. for every point $x \in A$ ) there exists a $G_{\delta}$-set $G$ in $X$ such that $H \subset G$ (resp. $x \in G$ ) and $G \cap A$ is closed in $X$. In a normal (resp. regular) space every generalized $G_{\delta}$ (resp. point-generalized $G_{\delta}$ ) is a pseudogeneralized $G_{\delta}$ (resp. pseudopoint-generalized $G_{\delta}$ ), but there exists a completely regular space with an open subset which is not a pseudogeneralized $G_{\delta}$ in the whole space (7.3).

It is easy to verify that a "transitive law" similar to 2.6 holds for pseudogeneralized $G_{\delta}$ and pseudopoint-generalized $G_{\delta}$-sets. There is, however, no result similar to 
2.5 because every closed set is a pseudogeneralized $G_{\delta}$. Thus, in a $T_{1}$-space every subset is a union of pseudogeneralized $G_{\delta}$-sets. We do get, however, the following result which is similar to 2.7 .

6.1. Proposition. Let $X$ be a Tychonoff space. The following are equivalent.

(a) For every compactification $(Y, c)$ of $X, c(X)$ is a pseudogeneralized $G_{\delta}$ (resp. pseudopoint-generalized $G_{\delta}$ ) in $Y$.

(b) $\beta(X)$ is a pseudogeneralized $G_{\delta}$ (resp. pseudopoint-generalized $G_{\delta}$ ) in $\beta X$.

(c) There exists a compactification $(Y, c)$ of $X$ such that $c(X)$ is a pseudogeneralized $G_{\delta}$ (resp. pseudopoint-generalized $G_{\delta}$ ) in $Y$.

Proof. The proof of $(c) \rightarrow(b)$ is straightforward. We prove (b) $\rightarrow$ (a) for the "pseudogeneralized" case. Let $f: \beta X \rightarrow Y$ be the continuous map such that $c=\beta \circ f$. Let $K \subset c(X)$ such that $K$ is closed in $Y$, then $f^{-1}(K) \subset \beta(X)$, and $f^{-1}(K)$ is closed in $\beta X$. By hypothesis, there exists a $G_{\delta}$-set $G=\bigcap_{i=1}^{\infty} G_{i}$ in $\beta X$ such that $f^{-1}(K) \subset G$ and $G \cap \beta(X)$ is closed in $\beta X$. Construct saturated open sets $S_{i}$ (i.e. each $S_{i}=f^{-1}\left(U_{i}\right)$ where $U_{i}$ is open in $Y$ ) in $\beta X$ such that $S_{0}=\beta X$, and $f^{-1}(K)$ $\subset S_{i} \subset \bar{S}_{i} \subset S_{i-1} \cap G_{i}$. Then $S=\bigcap_{i=1}^{\infty} S_{i}$ is a closed $G_{\delta}$ in $\beta X$ such that $K \subset f(S)$ $\subset f(G)$, and $f(S)$ is a closed $G_{\delta}$ in $Y$. Since $f$ is injective on $\beta(X)$ we have $f(S)$ $\cap c(X) \subset f(G \cap \beta(X)) \subset c(X)$, but $f(G \cap \beta(X))$ is closed in $Y$. Therefore, $f(S)$ $\cap c(X)$ is also closed in $Y$.

6.2. THEOREM. Let $X$ be a Tychonoff space.

(a) $X$ is of pseudocountable type if and only if $X$ is a pseudogeneralized $G_{\delta}$ in $\beta X$.

(b) $X$ is of pseudopoint-countable type if and only if $X$ is a pseudopoint-generalized $G_{\delta}$ in $\beta X$.

Proof. The proof follows from the definitions involved.

6.3. Proposition. Let $Y$ be a Tychonoff space of pseudocountable type (resp. pseudopoint-countable type), then every closed subset and every pseudogeneralized $G_{\delta}$ (resp. pseudopoint-generalized $G_{\delta}$ ) is a space of the same type as $Y$.

Proof. Since every closed set is a pseudogeneralized $G_{\delta}$ and a pseudopointgeneralized $G_{\delta}$ we need only show the latter result. To see that, we note that 5.1 (b) still holds for the "pseudo" case and then use 6.1 and 6.2.

Since Tychonoff spaces are regular, it is a corollary to 6.3 that being of pseudopoint-countable type is hereditary to point-generalized $G_{\delta}$-subsets. Unless $Y$ is normal, however, 6.3 does not tell us that generalized $G_{\delta}$-subsets of spaces of pseudocountable type are of the same type. To obtain that result (namely 6.5) we need the next lemma.

6.4. LEMMA. Let $X \subset Y \subset Z$, where $Z$ is a normal space. If $Y$ is a pseudogeneralized $G_{\delta}$ in $Z$, and $X$ is a generalized $G_{\delta}$ in $Y$, then $X$ is a pseudogeneralized $G_{\delta}$ in $Z$.

Proof. Let $H \subset X$, and $H$ closed in $Z$. By the hypothesis on $X$, there exists a $G_{\delta}$-set $G=\bigcap_{i=1}^{\infty} G_{i}$ in $Y$ such that $H \subset G \subset X$. Since $H$ is closed and $Z$ normal, 
we may assume that $G$ is closed in $Y$. By the hypothesis on $Y$, there exists a $G_{\delta}$-set $T$ in $Z$ such that $H \subset T$ and $T \cap Y$ is closed in $Z$. Let $G^{\prime}$ be a $G_{\delta}$ in $Z$ such that $G^{\prime} \cap Y=G$. Then $G^{\prime} \cap T$ is the desired $G_{\delta}$.

6.5. Proposition. Let $Y$ be a Tychonoff space of pseudocountable type. Then every generalized $G_{\delta}$ subset of $Y$ is a space of pseudocountable type.

Proof. The proof is similar to that of 5.2.

In light of 5.4 and 5.6, we remark that a Hausdorff space in which every closed set is a $G_{\delta}$ is a space of pseudocountable type, and a $T_{1}$-space in which every point is a $G_{\delta}$ is a space of pseudopoint-countable type.

Spaces of pseudocountable and pseudopoint-countable type behave in some ways similarly to the spaces of countable and point-countable type. For example, results 6.6 and 6.7 below together with analogous results by Arhangel'skil show that for Tychonoff spaces all four types are countably productive, and two are preserved and reflected by perfect (=proper) maps.

6.6. Proposition. Let $X_{i}$ be a space of pseudocountable (resp. pseudopointcountable) type for each $i \in N$. Then $\prod\left\{X_{i}: i \in N\right\}$ is of the same type.

Proof. This result follows from the fact that a countable product of compact $G_{\delta}$-sets is again a compact $G_{\delta}$.

6.7. Proposition. Let $f: X \rightarrow Y$ be a perfect map (surjection). If $Y$ is of pseudocountable type, then $X$ is of pseudocountable type. If $X$ is a regular space of pseudocountable type, then $Y$ is a regular space of pseudocountable type.

Proof. To prove the first assertion we need only note that if $H$ is a compact $G_{\delta}$ in $Y$, then $f^{-1}(H)$ is a compact $G_{\delta}$ in $X$. For the latter assertion, it is well known that under the given hypothesis, $Y$ is regular. If $H$ is a compact set in $Y$, then $f^{-1}(H)$ is contained in a compact $G_{\delta}$ in $X$. As in the proof of 6.1 we may assume that a compact $G_{\delta}$ is saturated, hence its image is a compact $G_{\delta}$ containing $H$.

7. Examples. Below we give the examples to which reference was made in $\$ 6$. In 7.4 an example of a first countable space which is not of pseudocountable type is given. Examples 7.1 and 7.2 show that these spaces of "pseudo" type are not related in general to $k$-spaces. Example 7.5 yields a solution to a problem posed by Arhangel'skil [2, Problem 3, p. 60], and settles the more general analogue for "pseudo" spaces.

7.1. ExAMPLE. A Tychonoff space of pseudocountable type which is not a $k$-space. Let $p \in \beta N-N$. The desired space is $X=N \cup\{p\}$ with the subspace topology induced from $\beta N$. Every closed set in $X$ is a $G_{\delta}$, and every compact set is finite.

7.2. EXAMPLE. A Tychonoff, $k$-space which is not of pseudopoint-countable type. The space $J$ in $[8]$ is the desired example. Let $[0, \Omega]$ be the set of ordinals 
less than or equal to the first uncountable ordinal. Let $X=[0, \Omega] \times N$. $J$ is the quotient space obtained by collapsing $\{(\Omega, n): n \in N\}$ to a point $j$. It is easy to see that the point $j \in J$ is not contained in any compact $G_{\delta}$.

7.3. EXAMPLE. An open subset of a Tychonoff space which is not a pseudogeneralized $G_{\delta}$ in the space. The desired space is the Tychonoff plank [4, p. 123] and the desired open set is the complement of the top (=uncountable) edge.

7.4. EXAMPLE. A first countable space which is not of pseudocountable type. Let $X$ be a space and $M \subset X$. By [3, Example 2, p. 215], sets of the form $U \cup K$ where $U$ is open in $X$ and $K \subset X-M$ define a topology on $X$. The resulting space $X_{M}$ has the property that every point in $X-M$ is isolated. In particular, let $X$ be the lexicographically ordered square, and $M$ the union of the top and bottom lines. The space $X_{M}$ is the desired example. If $H$ is a compact $G_{\delta}$ in $X_{M}$ which contains $M$, then $H=\bigcap_{i=1}^{\infty}\left(U_{i} \cup K_{i}\right)$ where $U_{i}$ is open in $X$ and $K_{i} \subset X-M$ for all $i$. Since $X-H \subset \bigcup_{i=1}^{\infty}\left(X-U_{i}\right)$, and $M \subset \bigcap_{i=1}^{\infty} U_{i}$, we have that $\bigcup_{i=1}^{\infty}\left(X-U_{i}\right)$ is contained in countably many vertical lines. Thus $H$ contains a vertical line, and it follows that $H$ contains a closed infinite discrete set. Hence $M$ is not contained in any compact $G_{\delta}$ in $X_{M}$. The space $D$ in 2.9 is also such an example.

7.5. EXAMPLE. The perfect image of a first countable space need not be of pointcountable type nor even of pseudopoint-countable type. Let $X_{M}$ be the space constructed in 7.4. The space obtained by collapsing $M$ to a point $m$ is the desired example. Clearly, the quotient map is perfect, and the quotient space is not of pseudopoint-countable type (at $m$ ).

Added in proof. At the Pittsburgh Topology Conference (1970), D. J. Lutzer presented an example which showed that the perfect image of a first countable space need not be of point-countable type, and he informed the author that the first such example was given by $\mathrm{M}$. M. Čoban [Perfect mappings and spaces of countable type, Vestnik Moskov. Univ. Ser. I Mat. Meh. 22 (1967), 87-93. MR 36, 3313]. Both of these examples have the feature that every point in the image space is a $G_{\delta}$.

\section{REFERENCES}

1. A. V. Arhangel'skiī, On a class of spaces containing all metric and all locally bicompact spaces, Dokl. Akad. Nauk SSSR 151 (1963), 751-754=Soviet Math. Dokl. 4 (1963), 1051-1055. MR 27 \#2959.

2. — Bicompact sets and the topology of spaces, Trudy Moskov. Mat. Obšč. 13 (1965), 3-55 = Trans. Moscow Math. Soc. 1965, 1-62. MR 33 \#3251.

3. R. Engelking, Outline of general topology, PWN, Warsaw, 1965; English transl., NorthHolland, Amsterdam and Interscience, New York, 1968. MR 36 \#4508; MR 37 \#5836.

4. L. Gillman and M. Jerison, Rings of continuous functions, The University Series in Higher Math., Van Nostrand, Princeton, N. J., 1960. MR 22 \#6994.

5. M. Henriksen and J. R. Isbell, Some properties of compactifications, Duke Math. J. 25 (1957), 83-105. MR 20 \#2689.

6. E. Michael, A note on paracompact spaces, Proc. Amer. Math. Soc. 4 (1953), 831-838. MR 15, 144. 
7. S. Mrówka, Some properties of Q-spaces, Bull. Acad. Polon. Sci. Cl. III 5 (1957), 947-950. MR 20 \#1967.

8. Keiô Nagami, $\Sigma$-spaces, Fund. Math. 65 (1969), 169-192.

9. W. Rudin, Homogeneity problems in the theory of Cech compactifications, Duke Math. J. 23 (1956), 409-419; correction, p. 633. MR 18, 324.

10. Ju. M. Smirnov, On normally disposed sets of normal spaces, Mat. Sb. 29 (71) (1951), 173-176; English transl., NASA Technical Transl. F-193. MR 13, 371.

11. S. Negrepontis, An example on realcompactifications, Arch. Math. (Basel) 20 (1969), 162-164. MR 39\#6265.

\section{UNIVERSity Of North Carolina,}

Chapel Hill, North Carolina 27514 